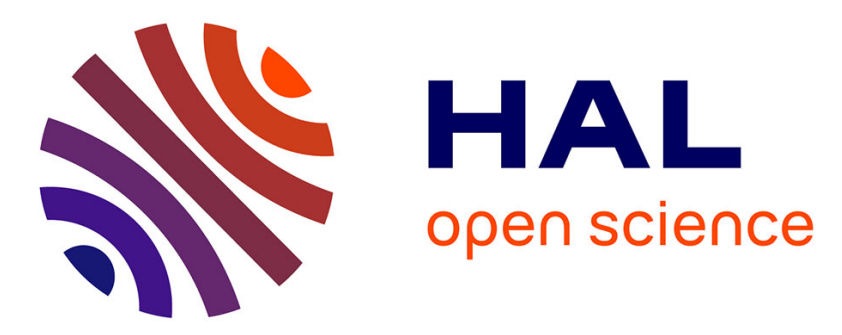

\title{
Development of an ultrafast electron source based on a cold-field emission gun for ultrafast coherent TEM
}

Giuseppe Mario Caruso, Florent Houdellier, Pierre Abeilhou, Arnaud Arbouet

\section{To cite this version:}

Giuseppe Mario Caruso, Florent Houdellier, Pierre Abeilhou, Arnaud Arbouet. Development of an ultrafast electron source based on a cold-field emission gun for ultrafast coherent TEM. Applied Physics Letters, 2017, 111 (2), pp.023101. 10.1063/1.4991681 . hal-01739806

\section{HAL Id: hal-01739806 https://hal.science/hal-01739806}

Submitted on 22 Mar 2018

HAL is a multi-disciplinary open access archive for the deposit and dissemination of scientific research documents, whether they are published or not. The documents may come from teaching and research institutions in France or abroad, or from public or private research centers.
L'archive ouverte pluridisciplinaire HAL, est destinée au dépôt et à la diffusion de documents scientifiques de niveau recherche, publiés ou non, émanant des établissements d'enseignement et de recherche français ou étrangers, des laboratoires publics ou privés. 


\section{Development of an ultrafast electron source based on a cold-field emission gun for ultrafast coherent TEM}

Giuseppe Mario Caruso, Florent Houdellier, Pierre Abeilhou, and Arnaud Arbouet

Citation: Appl. Phys. Lett. 111, 023101 (2017); doi: 10.1063/1.4991681

View online: https://doi.org/10.1063/1.4991681

View Table of Contents: http://aip.scitation.org/toc/apl/111/2

Published by the American Institute of Physics

\section{Articles you may be interested in}

As-grown two-dimensional $\mathrm{MoS}_{2}$ based photodetectors with naturally formed contacts

Applied Physics Letters 110, 261109 (2017); 10.1063/1.4990968

Peculiar torsion dynamical response of spider dragline silk

Applied Physics Letters 111, 013701 (2017); 10.1063/1.4990676

Low temperature equation of state of iron

Applied Physics Letters 111, 021903 (2017); 10.1063/1.4989688

Electrical switching of the magnetic vortex circulation in artificial multiferroic structure of $\mathrm{Co} / \mathrm{Cu} / \mathrm{PMN}-\mathrm{PT}(011)$ Applied Physics Letters 110, 262405 (2017); 10.1063/1.4990987

Chlorinated fluorine doped tin oxide electrodes with high work function for highly efficient planar perovskite solar cells

Applied Physics Letters 110, 263901 (2017); 10.1063/1.4989560

Electrode modulated capacitance-electric field nonlinearity in metal-insulator-metal capacitors

Applied Physics Letters 110, 263503 (2017); 10.1063/1.4989531

\section{Scilight}

Sharp, quick summaries illuminating the latest physics research

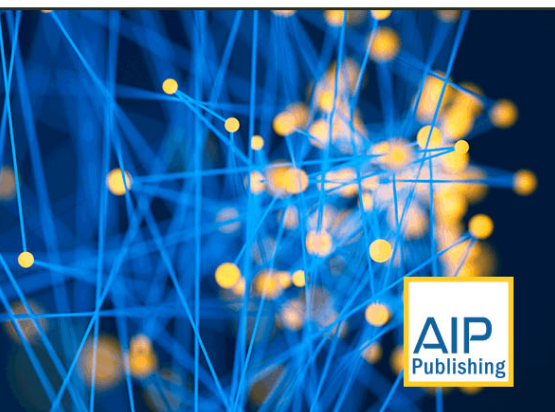




\title{
Development of an ultrafast electron source based on a cold-field emission gun for ultrafast coherent TEM
}

\author{
Giuseppe Mario Caruso, Florent Houdellier, ${ }^{\text {a) }}$ Pierre Abeilhou, and Arnaud Arbouet ${ }^{\text {b) }}$ \\ CEMES, CNRS UPR 8011 and University of Toulouse, 29 rue Jeanne Marvig, BP 94347, 31055 Toulouse, \\ France
}

(Received 20 March 2017; accepted 31 May 2017; published online 10 July 2017)

\begin{abstract}
We report on the design of a femtosecond laser-driven electron source for ultrafast coherent transmission electron microscopy. The proposed architecture allows introducing an ultrafast laser beam inside the cold field emission source of a commercial TEM, aligning and focusing the laser spot on the apex of the nanoemitter. The modifications of the gun assembly do not deteriorate the performances of the electron source in conventional DC mode and allow easy switching between the conventional and ultrafast laser-driven emission modes. We describe here this ultrafast electron source and discuss its properties. Published by AIP Publishing. [http://dx.doi.org/10.1063/1.4991681]
\end{abstract}

After the pioneering work by Bostanjoglo at TU Berlin, ultrafast Transmission Electron Microscopy (UTEM) has experienced a spectacular boost at the beginning of the century when A. Zewail at the California Institute of Technology demonstrated ultrafast TEM with both sub-picosecond time resolution and nanometer spatial resolution. ${ }^{1-3}$ In both cases, the ultrafast electron source was based on a flat photocathode illuminated by a pulsed laser. In the same way as for conventional TEMs, the potential of UTEMs is mostly governed by the properties of their electron source. Laser-driven flat photocathodes provide enough electrons for both single pulse and stroboscopic observation modes. However, electrons are emitted from a laser focal spot which is typically several tens of microns wide. This severely limits the brightness and spatial coherence of these electron sources and precludes their use for demanding applications such as coherent diffraction, high resolution spectroscopy, or electron holography. In conventional TEM, two kinds of electron sources based on metallic nano-emitters, Schottky Field Emission Guns (SFEG) and Cold-Field Emission guns (CFEG), are used when the brightness (i.e., the number of electrons emitted per unit solid angle and unit surface) is central to the targeted application. In DC Schottky electron sources, the nanotip is heated to enable its wetting by a layer of zirconia, and an extraction voltage is applied to the tip. The potential barrier is lowered by the Schottky effect enabling a significant fraction of the hot electron distribution to overcome the potential barrier and be emitted into vacuum. Schottky electron sources include a suppressor anode to confine the emission of the electrons at the apex of the tip. In cold-field emission guns, the monocrystalline oriented nanotip is not heated but placed in a high static electric field. When the applied E-field reaches a few $\mathrm{GV} / \mathrm{m}$, the potential barrier becomes thin enough to allow quantum mechanical tunneling of electrons into vacuum. Cold-field emission guns provide the best energy spread and brightness but their operation is by far the most demanding in terms of vacuum and electron optical properties. ${ }^{4}$ CFEGs do not have a suppressor anode.

\footnotetext{
a)florent.houdellier@cemes.fr

b)arnaud.arbouet@cemes.fr
}

The demonstration of ultrafast laser-driven electron emission from metallic nanotips has opened exciting perspectives for ultrafast electron microscopy. ${ }^{5-11}$ In particular, it has been demonstrated that the brightness of nanotip emitters is comparable in DC and ultrafast operation modes. ${ }^{12}$ Ultrafast electron emission from Schottky guns has first been implemented in Scanning Electron Microscopes. ${ }^{13,14}$ Then, the Schottky gun of a commercial TEM has been modified to trigger the electron emission with a femtosecond laser. ${ }^{15,16}$ The laser beam has been inserted inside the electron gun through an optical window routinely used to inspect the electron emission from outside the TEM gun. ${ }^{17}$ The laser beam is focused from outside the microscope through the optical window and redirected to the tip with an additional flat mirror. ${ }^{15,16}$ The availability of the suppressor anode is essential for the development of ultrafast electron sources based on Schottky guns as it allows to place the laser focusing optics outside the TEM gun. Indeed, a careful choice of the suppressor anode voltage compensates the loose focusing of the laser and confines the electron emission at the tip apex. The modification of a CFEG for ultrafast laser-driven operation in a TEM has not been reported yet.

Developing an ultrafast electron source based on a CFEG architecture with the laser focusing optics outside the TEM column will lead to a large emission region and deteriorate its properties. As a consequence, it is mandatory to insert the focusing optics inside the electron gun to minimize the size of the electron emission region and maximize the brightness of the source. However, the addition of optical and optomechanical components such as mirrors and mirror holder $(\mathrm{MH})$ close to the nanotip must carefully address issues related to vacuum and high voltage. In particular, the choice of the materials and their surface quality are critical. Finally, it is highly desirable that the modified electron source can be easily switched between the conventional DC mode for TEM alignment and routine sample characterization and the laser-driven ultrafast mode for time-resolved experiments. Finally, the modifications to the initial gun design must not deteriorate its potential for conventional TEM.

The developments described in this work have been achieved on the electron gun of a Hitachi HF2000 
CFEG-TEM with a modified vacuum system ${ }^{18-20}$ mounted on a dedicated UHV bench. In this work, we describe the design and main characteristics of the electron gun. Its performances for ultrafast TEM will be the subject of a forthcoming publication. Figure 1 shows the modifications to the original electron source. The accelerator is enclosed in a gun-housing filled with $\mathrm{SF}_{6}$ at a pressure of 2 bars for electrical insulation. Several parts of the original electron source have been modified. First, we have replaced the original gun-housing by another one which includes an optical window to allow a laser beam entering the $\mathrm{SF}_{6}$ region. We have developed a so-called optical head, which is an optical set-up allowing to visualize the nanotip, align the laser beam on the tip apex, and control the laser beam polarization from outside the electron source. The original architecture of the electron gun is shown in Fig. 1(c). The field-emission tip is a conventional commercial [310] oriented tungsten tip with an apex radius below $100 \mathrm{~nm}$. It is mounted on a metal/ceramic CF40 flange which insulates the nanotip from the extraction anode. The CF40 flange can be moved from outside the TEM column by a mechanical system (not shown for simplicity) to align the nanotip on the accelerator axis. The nanotip is placed at a potential $V_{0}\left(V_{0}=-200 \mathrm{kV}\right.$ in the present case) in a UHV environment $\left(P \sim 2 \times 10^{-9} \mathrm{~Pa}\right)$, a few millimeters away from the extraction anode which is at a potential $V_{1}$ relatively to $V_{0}$. A focusing anode (alternatively called gun-lens) at a potential $V_{2}$ relatively to $V_{0}$ is placed between the extraction anode and the accelerator. Adjusting the ratio between $V_{2}$ and $V_{1}$ allows adjusting the beam cross-over inside the acceleration tube. The details of the modified electron source are shown in Fig. 1(d). The optical head consists of an optical breadboard with optomechanical components enclosed in a box. The laser beam is first reflected by two mirrors mounted on piezo optical mounts. A polarizer and a half-wave plate allow controlling the polarization of the laser beam. The laser beam is sent into the electron gun after reflection on a beam splitter. It goes through a first optical window mounted on the gun-housing to enter the SF6 region. We have developed a customized version of the CF40 flange holding the nanotip which includes a $6 \mathrm{~mm}$ optical window. The laser beam enters the UHV region through this optical window and is then reflected by a plane mirror. It is finally focused by a parabolic mirror $(\mathrm{f}=8 \mathrm{~mm})$ inside a $\sim 3 \mu \mathrm{m}$ spot radius $\left(1 / e^{2}\right)$ at the apex of the nanotip. A mirror holder has been designed to hold the flat and parabolic mirrors in the immediate vicinity of the field emission (FE) tip. Reflective optics have been privileged to minimize the dispersion of the ultrafast pulses. The diameters of the flat and parabolic mirrors are, respectively, 8 and $6 \mathrm{~mm}$. The alignment of the mirror holder with respect to the tip is first realized outside the gun on a dedicated optical bench. The mirror holder is tightly held in position by a set of mirror polished screws and moves with the tip during the mechanical alignment of the electron beam with respect to the extractor hole. After this pre-alignment step, the CF40 flange with FE tip, mirror holder, and mirrors is placed back in the CFEG. The fine alignment of the laser beam on the tip apex is achieved by means of two piezo mirrors located in the optical head [see Fig. 1(d)]. The focusing of the laser beam on the tip is optimized by means of a telescope located outside the electron source. Figure 1(b) shows the beam of an He-Ne alignment laser focused on the tip apex. The mirrors, mirror holder and FE tip are at the same potential $\mathrm{V}_{0}$. The position of the laser spot is monitored using a CCD which collects the backscattered light. The

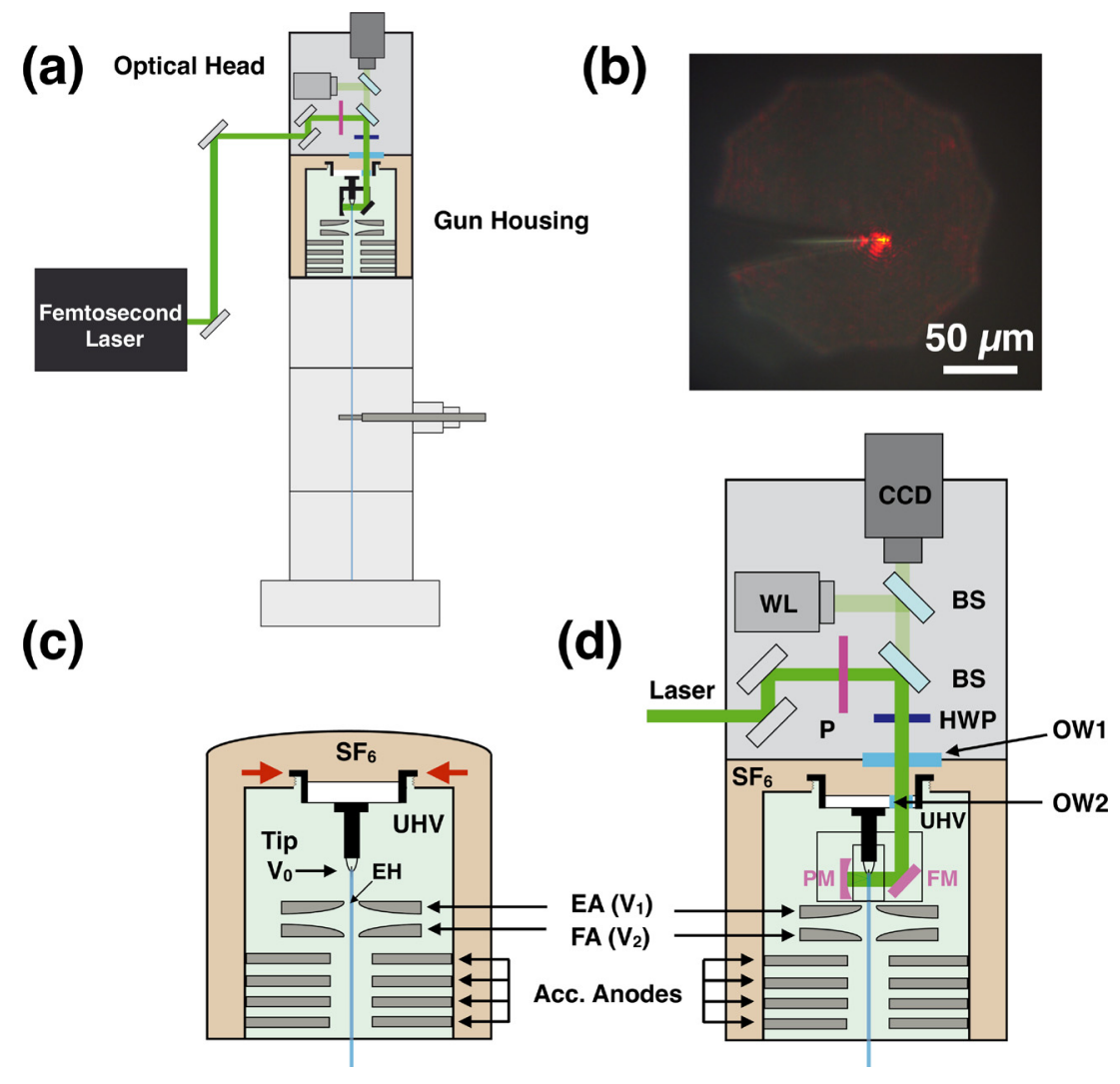

FIG. 1. (a) General design of the ultrafast CFE gun. An optical head with optical and optomechanical components is placed on top of the electron gun and allows to visualize the nanotip, optimize the laser beam alignment and focusing by means of remotely controlled motorized optomechanical components. (b) CCD image of a He$\mathrm{Ne}$ alignment laser focused on the nanotip. (c) Original scheme of the HF2000 CFE gun. EA: extraction anode. EH: extractor hole. FA: focusing anode. (d) Scheme of the ultrafast CFEG. WL: white light source, BS: optical beam-splitter, OW: optical window, FM: flat mirror, PM: parabolic mirror, HWP: half-wave plate, and P: polarizer. The mirror holder is cylindrically symmetric, the two mirrors being nested inside. 
optical head also includes a white light source to provide an image of the nanotip. The position of the laser beam and its polarization can be remotely controlled through a home-made control and diagnostics software. Extreme care has been paid to the surface quality of the optical components inserted in the nanotip region as the mirror holder is located at less than $2 \mathrm{~mm}$ from the extraction anode. All surfaces have been mirror polished until a surface roughness $\mathrm{Ra}=0.1 \mu \mathrm{m}$ was obtained. To warrant UHV operation conditions, the electron source must be baked at $350{ }^{\circ} \mathrm{C}$ during several hours. The mirror holder has been fabricated in the same material as the nanotip holder to minimize differential thermal expansion. It is important to notice that the original TEM column design has not been modified below the extraction anode to minimize the impact on the performance of the instrument.

We have performed numerical simulations to assess the influence of the mirror holder, introduced in the high voltage region close to the tip on the electron optical properties of the modified CFEG. Using the finite element method (FEM) software COMSOL multiphysics, ${ }^{22}$ we have computed the static field, with and without the mirror holder $(\mathrm{MH})$. A finer mesh has been used to accurately account for the tip apex geometry. For the same extraction voltage, the electric field at the apex is 2.5 times smaller with the MH compared to the original situation. In practice, this effect can be easily compensated by increasing the extraction voltage applied in standard DC operation. The $\mathrm{MH}$, being cylindrically symmetric, does not affect the symmetry of the electric field at the tip apex. Using SIMION 8.1, a finite difference method (FDM) based software, ${ }^{23}$ we have first cross-checked the previous results on the electric field and then computed electron trajectories with and without the mirror holder. As previously discussed, different mesh sizes have been used to best reproduce the gun geometry. Our calculations have consistently shown that the mirror holder shrinks the electron trajectories toward the source axis. This confinement can be clearly seen by comparing the left and right sides of Fig. 2. This effect changes the position of the virtual source of the gun assembly (tip, mirror holder and extraction anode $V_{1}$ ). However, the position of the virtual source of the gun can be easily adjusted independently using the focusing anode $V_{2}$ as in a standard CFEG. ${ }^{24}$ Combining these results with quantitative simulations, performed using Electron Optical Design (EOD), ${ }^{25}$ we have computed the optical properties of the gun with and without $\mathrm{MH}$ with a particular emphasis on its spherical aberration. Despite the modification of the electron trajectories described earlier, the overall aberration value is only slightly affected by the insertion of the mirror holder. Indeed, we get an aberration value of $35 \mathrm{~mm}$ (object side value) for $V_{1}=7 \mathrm{kV}$ and $V_{2}=35 \mathrm{kV}$ without the mirror holder, against $33 \mathrm{~mm}$ with mirror holder. This small difference is mainly due to a positive side-effect of the emission confinement by the mirror holder. Indeed, the latter decreases the incoming angle of the electron cone inside the focusing anode and, as a consequence, minimizes the contribution of this lens to the global spherical aberration. To summarize, our simulations clearly show that the mirror holder does not have any detrimental influence on the optical properties of the ultrafast electron source. It only affects the magnitude of the electric field at the apex. This effect can be compensated and a proper DC field emission can be recovered simply by adjusting the extraction voltage $V_{1}$. We have systematically acquired Fowler-Nordheim $\mathrm{I}\left(V_{1}\right)$ curves. Our experimental results confirm the reduction of the E-field at the tip apex predicted by the numerical simulations: whereas the onset for DC field-emission is around $2 \mathrm{kV}$ for the original CFEG, it is around $5.2 \mathrm{kV}$ for the modified one (for currents in the pA range).

We finally describe the laser-driven operation of the modified CFEG. Laser-driven emission of electrons from metallic nanotips has been investigated in different regimes both experimentally and theoretically. ${ }^{8-11}$ Laser-assisted electron emission from metallic surfaces can be obtained via several mechanisms. The single photon photoelectric effect involves the absorption of one incident photon of energy larger than the workfunction of the emitting surface. For photon energies smaller than the metal workfunction, electron emission is not possible by absorption of a single photon but becomes possible via the absorption of several photons at high optical excitation intensities. Larger intensities can lead to above-threshold photoemission in which a number of (a)

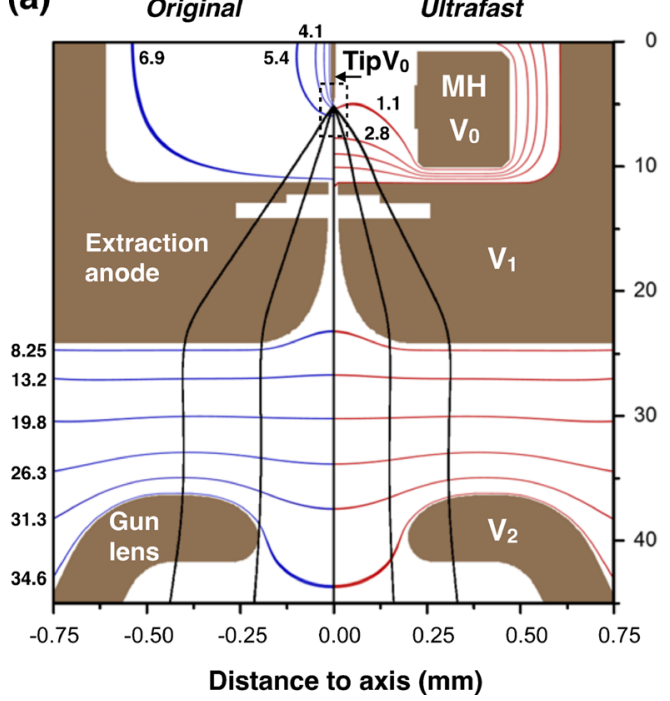

(b)

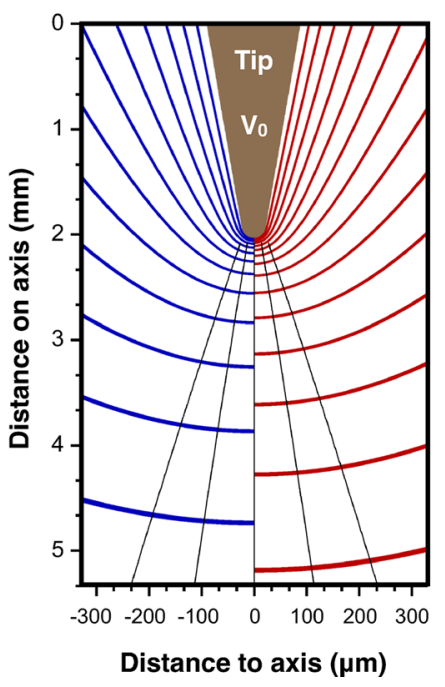

FIG. 2. (a) Electrons trajectories simulations performed using Simion 8.1 under the same conditions described in Kubo et al. ${ }^{21} \mathrm{~A}$ total of 5 electrons trajectories are compared between the original HF2000 gun assembly and the modified one to enable ultrafast laser-driven emission (computation shown with $V_{1}=7 \mathrm{kV}$ and $V_{2}=35 \mathrm{kV}$ ). Equipotential lines are also displayed using the same values for both sides enhancing the effect of the mirror holder. The values of the potentials are indicated on the left in $\mathrm{kV}$. (b) Magnified view of the tip region. 
photons larger than required from energy conservation are absorbed. ${ }^{8}$ Finally, even higher laser intensities can lead to optical field emission in which the optical fields are strong enough to bend the potential barrier. ${ }^{9}$ The efficiency of these emission processes is maximum for a polarization of the incident electric field normal to the metal surface. Our experiments have been performed using a compact ultrafast fiber laser, delivering ultrashort (350 fs), high energy (up to $20 \mu \mathrm{J}$ ), high repetition rate pulses at $1030 \mathrm{~nm}$ (Satsuma, Amplitude Systèmes). Electron emission has been obtained at a repetition rate of $1 \mathrm{MHz}$ using the femtosecond pulses generated at $515 \mathrm{~nm}$ by Second Harmonic Generation of the laser output in a beta barium borate (BBO) crystal. The laser power incident on the optical window of the CF40 flange was kept in the $0.5-4 \mathrm{~mW}$ range. The emitted current has been measured using an electrometer. We have first investigated the dependence of the electron yield on the laser power, extraction voltage $V_{1}$ and polarization. The emitted current, in the 0-7 pA range, increases with the incident laser power and extraction voltage [see Fig. 3(a)]. As can be seen in Fig. 3(a), the dark current of the high voltage system, measured at zero laser power, increases with the extraction voltage $V_{1}$. Furthermore, it is important to notice that the extraction voltage applied in these laser-driven emission experiments lies below the onset of DC field emission (around $5.2 \mathrm{kV}$ as mentioned earlier). In agreement with the established literature, our results show that the tip can be divided into two distinct regions with specific behaviours. As shown in the inset of Fig. 3(a), when the laser is focused exactly on the tip apex (later called the tip region), a

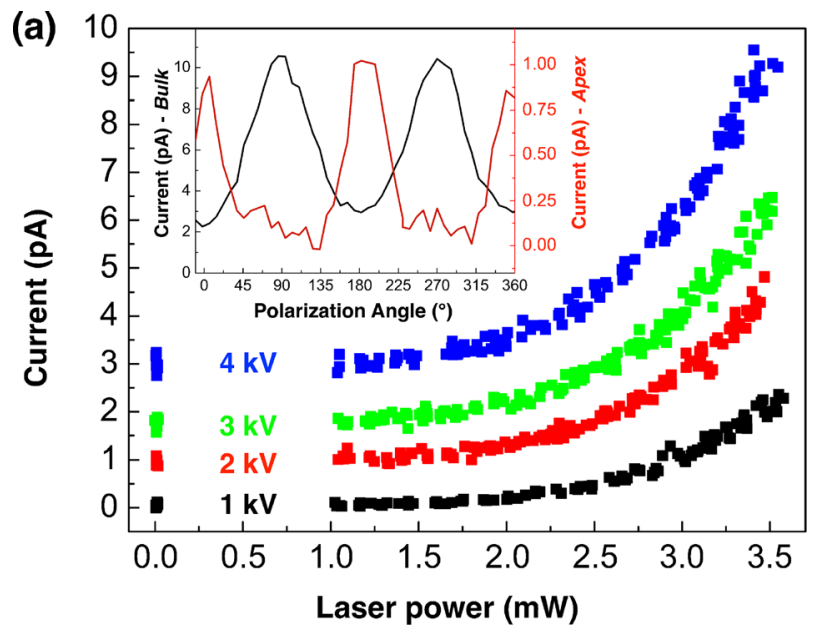

(b)

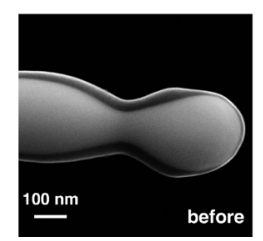

(c)

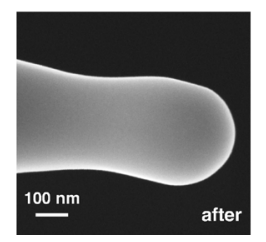

FIG. 3. (a) Electron yield measured at the tip apex as a function of the incident laser power for different values of the extraction voltage $V_{1}$. Inset: Dependence of the electron yield on the polarization of the laser electric field (at $0^{\circ}$ the laser E-field is parallel to the tip axis. (b) SEM image of a typical field-emission tip which has been routinely used in a CFEG-TEM in conventional mode. (c) SEM image of one of the nanotips used for several months for laser-driven electron emission. The noise of the high voltage system is visible at zero laser power where the result of several current measurements is shown. maximum electron yield is obtained for an incident polarization parallel with the tip axis. On the contrary, when the laser is focused a few microns away from the apex (later called the bulk region), the electron yield is maximum for an incident polarization perpendicular to the tip axis. Furthermore, the electron yield is larger in the bulk region than in the tip region as expected from the difference in metal surface contributing to the emission. In both regions, the power dependence plotted on a log-log scale has a slope slightly larger than 2. A strictly quadratic dependence would be expected from the energy of the incident photons $(2.41 \mathrm{eV})$ and the tungsten (310) facets work function $(4.36 \mathrm{eV})$. We have carefully checked that the electron yield was proportional to the repetition rate of the laser. This confirmed that cumulative heating effects have a negligible influence in our experimental conditions. We have taken scanning electron microscopy (SEM) images of the tip after the laser-driven emission experiments. Post-mortem analysis of several tips has shown that the size of the apex is slightly larger after the experiments. This reshaping is probably due to the transient heating of the nanotip induced by single laser pulses at the highest laser powers. Indeed, under these conditions, approximately 40 electrons are emitted per laser pulse which is close to the damage threshold of tungsten nanotips. ${ }^{26}$ The detailed study of these effects will be the subject of a forthcoming publication.

In summary, we have reported on the development of an ultrafast electron source based on a modified commercial cold field emission source. This ultrafast electron source architecture allows easy switching between conventional DC- and ultrafast laser driven emission modes. Electron optical simulations show that the modifications brought to the original CFEG design have a limited influence on the optical properties of the electron source and should not deteriorate significantly the TEM performances. Laser-driven electron emission has been investigated and demonstrated that the electron yield can be controlled through the incident laser power and polarization and the extraction voltage. Further investigations are under progress to assess the performances of this ultrafast electron source for TEM applications.

The authors thank the Institut de Physique du CNRS and Agence Nationale de la Recherche for financial support (ANR Grant No. ANR-14-CE26-0013). This work was supported by Programme Investissements d'Avenir under the Program ANR-11-IDEX-0002-02, reference ANR-10LABX-0037-NEXT (MUSE grant). This work was supported by the computing facility center CALMIP of the University Paul Sabatier of Toulouse France. The authors acknowledge financial support from the European Union under the Seventh Framework Program under a contract for an Integrated Infrastructure Initiative (Reference 312483-ESTEEM2). The authors are grateful to E. Snoeck, M. J. Hÿtch, and M. Kociak for their support. The authors acknowledge B. Chalopin and S. Weber for fruitful discussions.

${ }^{1}$ W. E. King, M. R. Armstrong, O. Bostanjoglo, and B. W. Reed, "Highspeed electron microscopy," in Science of Microscopy, edited by P. W. Hawkes and J. C. H. Spence (Springer, New York, NY, 2007), pp. 406-444.

${ }^{2}$ V. A. Lobastov, R. Srinivasan, and A. H. Zewail, Proc. Natl. Acad. Sci. U. S. A. 102, 7069 (2005). 
${ }^{3}$ A. H. Zewail and J. M. Thomas, $4 d$ Electron Microscopy: Imaging in Space and Time (Imperial College Press, 2009).

${ }^{4}$ A. V. Crewe, D. N. Eggenberger, J. Wall, and L. M. Welter, Rev. Sci. Instrum. 39, 576 (1968).

${ }^{5}$ P. Hommelhoff, Y. Sortais, A. Aghajani-Talesh, and M. A. Kasevich, Phys. Rev. Lett. 96, 077401 (2006).

${ }^{6}$ P. Hommelhoff, C. Kealhofer, and M. A. Kasevich, Phys. Rev. Lett. 97, 247402 (2006).

${ }^{7}$ B. Barwick, C. Corder, J. Strohaber, N. Chandler-Smith, C. Uiterwaal, and H. Batelaan, New J. Phys. 9, 142 (2007).

${ }^{8}$ M. Schenk, M. Krüger, and P. Hommelhoff, Phys. Rev. Lett. 105, 257601 (2010).

${ }^{9}$ R. Bormann, M. Gulde, A. Weismann, S. V. Yalunin, and C. Ropers, Phys. Rev. Lett. 105, 147601 (2010).

${ }^{10}$ D. J. Park, B. Piglosiewicz, S. Schmidt, H. Kollmann, M. Mascheck, P. Groß, and C. Lienau, Ann. Phys. 525, 135 (2013).

${ }^{11}$ S. Thomas, M. Krüger, M. Förster, M. Schenk, and P. Hommelhoff, Nano Lett. 13, 4790 (2013).

${ }^{12}$ D. Ehberger, J. Hammer, M. Eisele, M. Krüger, J. Noe, A. Högele, and P. Hommelhoff, Phys. Rev. Lett. 114, 227601 (2015).

${ }^{13}$ D.-S. Yang, O. F. Mohammed, and A. H. Zewail, Proc. Natl. Acad. Sci. U. S. A. 107, 14993 (2010).
${ }^{14}$ O. F. Mohammed, D.-S. Yang, S. K. Pal, and A. H. Zewail, J. Am. Chem. Soc. 133, 7708 (2011).

${ }^{15}$ R. Bormann, S. Strauch, S. Schäfer, and C. Ropers, J. Appl. Phys. 118, 173105 (2015).

${ }^{16}$ A. Feist, K. E. Echternkamp, J. Schauss, S. V. Yalunin, S. Schäfer, and C. Ropers, Nature 521, 200 (2015).

${ }^{17}$ K. Hojo, H. Kurata, and T. Tomita, Japan patent JP2006114260 (A) (2011).

${ }^{18} \mathrm{H}$. Murakoshi, M. Ichihashi, S. Isakozawa, and Y. Sato, U.S. patent $5,134,289$ (1992).

${ }^{19}$ F. Houdellier, L. de Knoop, C. Gatel, A. Masseboeuf, S. Mamishin, Y. Taniguchi, M. Delmas, M. Monthioux, M. Hýtch, and E. Snoeck, Ultramicroscopy 151, 107 (2015).

${ }^{20}$ A. Arbouet and F. Houdellier, U.S. patent 9263229 (2016).

${ }^{21}$ Y. Kubo, C. Gatel, E. Snoeck, and F. Houdellier, Ultramicroscopy 175, 67 (2017).

${ }^{22}$ See https://www.comsol.fr for information about the FEM software COMSOL.

${ }^{23}$ D. A. Dahl, Int. J. Mass Spectrom. 200, 3 (2000).

${ }^{24}$ H. M. Hachioji, M. Kodaira, S. Isakozawa, and Y. Yato, U.S. patent n005134289A (1992).

${ }^{25}$ B. Lencová and J. Zlámal, Phys. Procedia 1, 315 (2008).

${ }^{26}$ S. A. Hilbert, A. Neukirch, C. J. G. J. Uiterwaal, and H. Batelaan, J. Phys. B 42, 141001 (2009). 